\title{
INTEGRABILITY OF THE RUCKLIDGE SYSTEM
}

\author{
MAURÍCIO F. S. LIMA ${ }^{1}$, JAUME LLIBRE ${ }^{2}$ AND CLÀUDIA VALLS ${ }^{3}$
}

\begin{abstract}
We study the Darboux and the analytic integrability of the Rucklidge system.
\end{abstract}

\section{Introduction AND STATEMENT OF THE MAIN RESUlts}

The Rucklidge system (see [9]):

$$
\begin{aligned}
& \dot{x}=-a x+b y-y z, \\
& \dot{y}=x, \\
& \dot{z}=-z+y^{2},
\end{aligned}
$$

is a famous model (see for instance [9]), where $x, y, z \in \mathbb{R}^{3}$ are the state variables, $(a, b)$ are real parameters and the dot denotes the derivative with respect to the time $t$. This model considers the problem of two-dimensional convection in a horizontal layer of Boussinesq fluid with lateral constants. It provides an accurate description of convection in the parameter regime where the chaotic solutions appear. Despite its simplicity it has a reach local dynamical behavior as chaotic attractors for some values of the parameters $a$ and $b$ (for example when $a=2, b=6.7$ or $a=-0.1$ and $b=-1$ ) and has been widely analyzed (see for instance $[10,11]$ and the references therein).

We note that system (1) is a family of quadratic systems in a three dimensional space. Quadratic systems in $\mathbb{R}^{3}$ are the simplest systems after the linear ones. Examples of such systems are the well-known Lorenz system, Rössler system, Rikitake system, among others. These have been investigated in the last decades from different dynamical points of view. Despite their simplicity quadratic systems are not completely understood from the view point of the integrability, see for instance [8].

The aim of this paper is to study the existence of Darboux and analytic first integrals of system (1). We recall that a first integral of Darboux type is a first integral $H$ which is a function of Darboux type (see below (2) for a precise definition). The study of the integrability is a classical problem in the theory of differential equations.

The vector field associated to system (1) is

$$
\mathbf{X}=(-a x+b y-y z) \frac{\partial}{\partial x}+x \frac{\partial}{\partial y}+\left(-z+y^{2}\right) \frac{\partial}{\partial z} .
$$

Let $U \subset \mathbb{C}^{3}$ be an open set. We say that the non-constant function $H: U \rightarrow \mathbb{C}$ is a first integral of the polynomial vector field $\mathbf{X}$ on $U$ if $H(x(t), y(t), z(t))$ is constant

2010 Mathematics Subject Classification. Primary 34C05, 34A34, 34C14.

Key words and phrases. Analytic first integrals, Darboux first integrals, Darboux polynomials, exponential factors, averaging theory. 
for all values of $t$ for which the solution $(x(t), y(t), z(t))$ of $\mathbf{X}$ is defined on $U$. Clearly $H$ is a first integral of $\mathbf{X}$ on $U$ if and only if

$$
\mathbf{X} H=(-a x+b y-y z) \frac{\partial H}{\partial x}+x \frac{\partial H}{\partial y}+\left(-z+y^{2}\right) \frac{\partial H}{\partial z}=0 .
$$

on $U$.

In this paper we want to study the so-called Darboux first integrals of the polynomial differential systems (1), using the Darboux theory of integrability (originated in the papers [2]). For a present state of this theory see the Chapter 8 of [3], the paper [5], and the references quoted in them. Moreover, we also study the analytic integrability of system (1), i.e., the existence of a global analytic first integral $H: \mathbb{R}^{3} \rightarrow \mathbb{R}$.

We emphasize that the study of the existence of first integrals is a classical problem in the theory of differential systems, because the knowledge of first integrals of a differential system can be very useful in order to understand and simplify the topological structure of their orbits. Thus, their existence or not can also be viewed as a measure of the complexity of a differential system.

We recall that a first integral is of Darboux type if it is of the form

$$
f_{1}^{\lambda_{1}} \cdots f_{p}^{\lambda_{p}} F_{1}^{\mu_{1}} \cdots F_{q}^{\mu_{q}}
$$

where $f_{1}, \ldots, f_{p}$ are Darboux polynomials (see section 2 for a definition), $F_{1}, \ldots, F_{q}$ are exponential factors (see section 2 for a definition), and $\lambda_{j}, \mu_{k} \in \mathbb{C}$ for all $j$ and $k$.

The functions of the form (2) are called Darboux functions, and they are the base of the Darboux theory of integrability, which looks when these functions are first integrals or integrating factors. In this last case, the first integrals associated to integrating factors given by Darboux functions are the Liouvillian first integrals, see for more details $[3,5]$.

The Darboux theory of integrability is essentially an algebraic theory of integrability based in the invariant algebraic hypersurfaces that a polynomial differential system has. In fact to every Darboux polynomial there is associated some invariant algebraic hypersurface (see again section 2), and the exponential factors appear when an invariant algebraic surface has multiplicity larger than 1, for more details see $[1,3,5]$. As far as we know is the unique theory of integrability which is developed for studying the first integrals of polynomial differential systems. In general the other theories of integrability do not need that the differential system be polynomial.

The main results related to the integrability problem are summarized in the next theorems.

Theorem 1. System (1) has no Darboux polynomials.

As a straightforward consequence of this result we have the following result.

Corollary 2. System (1) has no polynomial first integrals or invariant algebraic surfaces.

We prove Theorem 1 and Corollary 2 in Section 3.

The next two results states the no existence of Darboux first integrals and analytic first integrals (for a set of the values of the parameters $(a, b)$ ) for system (1). 
Theorem 3. System (1) has no Darboux first integrals.

Theorem 4. System (1) has no global analytic first integral except perhaps in a set $S$ of zero Lebesgue measure in the plane of parameters $(a, b)$. The set $S$ is contained in a countable set of segments.

Remark 5. A more precise statement of Theorem 4 is given in Theorem 13.

We prove Theorem 3 in Section 3 and Theorem 13 in Section 4. Since the Darboux theory of integrability of a polynomial differential system is based on the existence of Darboux polynomials and their multiplicity, the study of the existence or not of Darboux first integrals needs to look for the Darboux polynomials. So the main steps for proving Theorem 3 are Theorem 10 and 1.

\section{BAsic Results}

Let $h=h(x, y, z) \in \mathbb{C}[x, y, z] \backslash \mathbb{C}$. As usual $\mathbb{C}[x, y, z]$ denotes the ring of all complex polynomials in the variables $x, y, z$. We say that $h$ is a Darboux polynomial of system (1) if it satisfies

$$
\mathbf{X} h=K h,
$$

the polynomial $K=K(x, y, z) \in \mathbb{C}[x, y, z]$ is called the cofactor of $h$ and has degree at most one. Every Darboux polynomial $h$ defines an invariant algebraic hypersurface $h=0$, i.e., if a trajectory of system (1) has a point in $h=0$, then the whole trajectory is contained in $h=0$, see for more details [3]. When $K=0$ the Darboux polynomial $h$ is a polynomial first integral.

We recall the following auxiliary result that was proved in [1].

Lemma 6. Let $f$ be a polynomial and $f=\prod_{j=1}^{s} f_{j}^{\alpha_{j}}$ its decomposition into irreducible factors in $\mathbb{C}[x, y, z]$. Then $f$ is a Darboux polynomial if and only if all the $f_{j}$ are Darboux polynomials. Moreover, if $K$ and $K_{j}$ are the cofactors of $f$ and $f_{j}$, then $K=\sum_{j=1}^{s} \alpha_{j} K_{j}$

An exponential factor $E$ of system (1) is a function of the form $E=\exp (g / h) \notin \mathbb{C}$ with $g, h \in \mathbb{C}[x, y, z]$ satisfying $(g, h)=1$ and

$$
\mathbf{X} E=L E
$$

for some polynomial $L=L(x, y, z)$ of degree at most one, called the cofactor of $E$.

A geometrical meaning of the notion of exponential factor is given by the next result.

Proposition 7. If $E=\exp (g / h)$ is an exponential factor for the polynomial differential system (1) and $h$ is not a constant polynomial, then $h=0$ is an invariant algebraic hypersurface, and eventually $e^{g}$ can be exponential factors, coming from the multiplicity of the infinite invariant hyperplane.

The proof of Proposition 7 can be found in $[1,6]$. We explain a little the last part of the statement of Proposition 7. If we extend to the projective space $\mathbb{P R}^{3}$ the polynomial differential system (1) defined in the affine space $\mathbb{R}^{3}$, then the hyperplane at infinity always is invariant by the flow of the extended differential system. 
Moreover, if this invariant hyperplane has multiplicity higher than 1 , then it creates exponential factors of the form $e^{g}$, see for more details [6].

Theorem 8. Suppose that the polynomial vector field $\mathbf{X}$ of degree $m$ defined in $\mathbb{C}^{4}$ admits $p$ invariant algebraic hypersurfaces $f_{i}=0$ with cofactors $K_{i}$, for $i=1, \ldots, p$ and $q$ exponential factors $E_{j}=\exp \left(g_{j} / h_{j}\right)$ with cofactors $L_{j}$, for $j=1, \ldots, q$. Then there exists $\lambda_{i}, \mu_{j} \in \mathbb{C}$ not all zero such that

$$
\sum_{i=1}^{p} \lambda_{i} K_{i}+\sum_{j=1}^{q} \mu_{j} L_{j}=0
$$

if and only if the function of Darboux type

$$
f_{1}^{\lambda_{1}} \cdots f_{p}^{\lambda_{p}} E_{1}^{\mu_{1}} \cdots E_{q}^{\mu_{q}}
$$

is a first integral of $\mathbf{X}$.

Theorem 8 is proved in [3]. The following result is well-known.

Lemma 9. Assume that $\exp \left(g_{1} / h_{1}\right), \ldots, \exp \left(g_{r} / h_{r}\right)$ are exponential factors of some polynomial differential system

$$
x^{\prime}=P(x, y, z), y^{\prime}=Q(x, y, z), z^{\prime}=R(x, y, z),
$$

with $P, Q, R \in \mathbb{C}[x, y, z]$ with cofactors $L_{j}$ for $j=1, \ldots, r$. Then

$$
\exp (G)=\exp \left(g_{1} / h_{1}+\cdots+g_{r} / h_{r}\right)
$$

is also an exponential factor of system (3) with cofactor $L=\sum_{j=1}^{r} L_{j}$.

\section{Proof of Theorem 1 and Theorem 3.}

We consider the automorphism $\tau: \mathbb{C}^{3}[x, y, z] \rightarrow \mathbb{C}^{3}[x, y, z]$ defined by

$$
\tau(p(x, y, x))=p(-x,-y, z) .
$$

The following result characterizes the polynomial first integrals of system (1), i.e. it characterizes the Darboux polynomial with zero cofactor that are invariant by $\tau$.

Theorem 10. There are no polynomial first integrals of system (1) that are invariant by $\tau$.

Proof. Let $h=h(x, y, z)$ be a polynomial first integral of system (1) that is invariant by $\tau$. We write it as a sum of homogeneous polynomials as

$$
h=\sum_{j=0}^{n} h_{j}(x, y, z)
$$

where each $h_{j}$ is a homogeneous polynomial of degree $j$. Without loss of generality we can assume that $h_{0}=0, n>0$ and $h_{n} \neq 0$. Note that if $h$ is a polynomial first integral then

$$
(-a x+b y-y z) \frac{\partial h}{\partial x}+x \frac{\partial h}{\partial y}+\left(-z+y^{2}\right) \frac{\partial h}{\partial z}=0 .
$$


Now we define the following linear partial differential operator $L:=-y z \frac{\partial}{\partial x}+$ $y^{2} \frac{\partial}{\partial z}$. Computing the coefficient of degree $n+1$ in (4) we get

$$
L\left(h_{n}\right)=-y z \frac{\partial h_{n}}{\partial x}+y^{2} \frac{\partial h_{n}}{\partial z}=0 .
$$

To solve system (5) is equivalent to look for a polynomial first integral of system

$$
\dot{x}=-y z, \quad \dot{y}=0, \quad \dot{z}=y^{2} .
$$

We introduce the change of variables

$$
Y=y, \quad Z=z, \quad X=x y+\frac{z^{2}}{2}
$$

with inverse

$$
y=Y, \quad z=Z, \quad x=\frac{X-Z^{2} / 2}{Y}
$$

Then we can rewrite system (6) as

$$
\dot{X}=0, \quad \dot{Y}=0, \quad \dot{Z}=Y^{2}
$$

which implies that system (5) becomes

$$
Y^{2} \frac{\partial \tilde{h}_{n}}{\partial Z}=0
$$

where $\tilde{h}_{n}=\tilde{h}_{n}(X, Y, Z)=h_{n}(x, y, z)$. Clearly

$$
\tilde{h}_{n}=\tilde{h}_{n}(Y, X)=\sum_{l+2 k=n} a_{l, k} Y^{l} X^{k}=\sum_{l+2 k=n} a_{l, k} y^{l}\left(x y+z^{2} / 2\right)^{k} .
$$

Since $h_{n}$ is invariant by $\tau$ we must have $\tau\left(h_{n}\right)=h_{n}$ that is

$$
\sum_{l+2 k=n}(-1)^{l} a_{l, k} y^{l}\left(x y+z^{2} / 2\right)^{k}=\sum_{l+2 k=n} a_{l, k} y^{l}\left(x y+z^{2} / 2\right)^{k} .
$$

Hence, $a_{l, k}=0$ for $l$ odd. This yields that $l$ must be even and thus

$$
h_{n}=\sum_{2 l+2 k=n} a_{l, k} y^{2 l}\left(x y+z^{2} / 2\right)^{k} .
$$

Then $n=2 m$ that is $l+k=m$ with

$$
h_{2 m}=\sum_{l=0}^{m} a_{l, m-l} y^{2 l}\left(x y+z^{2} / 2\right)^{m-l}=\sum_{l=0}^{m} b_{l} y^{2 l}\left(x y+z^{2} / 2\right)^{m-l},
$$

where $b_{l}=a_{l, m-l}$. Note that

$$
\tilde{h}_{2 m}=\sum_{l=0}^{m} b_{l} Y^{2 l} X^{m-l} .
$$

Now we will show by induction that for $j=1, \ldots, m$,

$$
\tilde{h}_{2 m-j}=\sum_{r=0}^{[j / 2]} \sum_{l=2(j-2 r)}^{m-r} c_{l, r} X^{m-l+j-3 r}\left(\frac{(-1)^{j-2 r}}{(j-2 r) !} A_{j} Y^{2 l-4(j-2 r)} Z^{j-2 r}+O\left(X^{-1}\right)\right),
$$


where

$$
A_{j}=\prod_{s=1}^{j-2 r}(m+l-r-3(s-1)), \quad c_{l, 0}=b_{l},
$$

and $O\left(X^{-1}\right)$ denotes polynomials in the variable $X^{-1}$ (with coefficients depending in the variables $Y, Z)$.

We compute the terms of order $n=2 m$ in (4) we get

$$
L\left(h_{n-1}\right)=(a x-b y) \frac{\partial h_{n}}{\partial x}-x \frac{\partial h_{n}}{\partial y}+z \frac{\partial h_{n}}{\partial z}
$$

which is equivalent with the change of variables in (7) as

$$
\begin{aligned}
Y^{2} \frac{\partial \tilde{h}_{n-1}}{\partial Z}= & \left(\frac{a}{Y}\left(X-\frac{Z^{2}}{2}\right)-b Y\right) \frac{\partial \tilde{h}_{n}}{\partial X} \frac{\partial X}{\partial x}-\left(\frac{X-Z^{2} / 2}{Y}\right)\left(\frac{\partial \tilde{h}_{n}}{\partial Y}+\frac{\partial \tilde{h}_{n}}{\partial X} \frac{\partial X}{\partial y}\right) \\
& +Z \frac{\partial \tilde{h}_{n}}{\partial X} \frac{\partial X}{\partial z}=\left(a\left(X-Z^{2} / 2\right)-b Y^{2}\right) \frac{\partial \tilde{h}_{n}}{\partial X}+Z^{2} \frac{\partial \tilde{h}_{n}}{\partial X} \\
& -\left(\frac{X-Z^{2} / 2}{Y}\right) \frac{\partial \tilde{h}_{n}}{\partial Y}-\frac{1}{Y^{2}}\left(X^{2}-X Z^{2}+Z^{4} / 4\right) \frac{\partial \tilde{h}_{n}}{\partial X}+Z \frac{\partial \tilde{h}_{n}}{\partial Z} .
\end{aligned}
$$

Note that in the right hand of equation (11) the parts that will provide the highest degree in $X$ are

$$
-\frac{X}{Y} \frac{\partial \tilde{h}_{n}}{\partial Y}-\frac{X^{2}}{Y^{2}} \frac{\partial \tilde{h}_{n}}{\partial X}
$$

so we only need to consider them. Hence, using (9) we obtain

$$
\begin{aligned}
\frac{\partial \tilde{h}_{n-1}}{\partial Z} & =-\sum_{l=0}^{m} b_{l} X^{m-l+1}\left((2 l+(m-l)) Y^{2 l-4}+O\left(X^{-1}\right)\right) \\
& =-\sum_{l=0}^{m} b_{l} X^{m-l+1}\left((m+l) Y^{2 l-4}+O\left(X^{-1}\right)\right) .
\end{aligned}
$$

Integrating it with respect to $Z$ we obtain

$$
\tilde{h}_{n-1}=-\sum_{l=0}^{m} b_{l} X^{m-l+1}\left((m+l) Y^{2 l-4} Z+O\left(X^{-1}\right)\right)+\tilde{g}_{n-1}(Y, X) .
$$

Since $\tilde{h}_{n-1}$ must be invariant by $\tilde{\tau}(p(X, Y, Z))=p(X,-Y, Z)$ and must have dimension $2 m-1$ we get that $\tilde{g}_{n-1}(X, Y)=\sum_{2 l+2 k=2 m-1} c_{l} Y^{2 l} X^{k}$ which is not possible and thus $\tilde{g}_{n-1}=0$. Hence,

$$
\tilde{h}_{n-1}=-\sum_{l=0}^{m} b_{l} X^{m-l+1}\left((m+l) Y^{2 l-4} Z+O\left(X^{-1}\right)\right) .
$$

Since $\tilde{h}_{n-1}$ is a polynomial we must have $b_{l}=0$ for $l=0,1$ and thus

$$
\tilde{h}_{n-1}=-\sum_{l=2}^{m} b_{l} X^{m-l+1}\left((m+l) Y^{2 l-4} Z+O\left(X^{-1}\right)\right) .
$$

which satisfies (10) for $j=1$ with $c_{l, 0}=b_{l}$. 
Now assume (10) is true for $j=1, \ldots, k$ and we will prove it for $j=k+1$. Hence if we compute the terms of order $n-k$ in (4) we get

$$
L\left(h_{n-k-1}\right)=(a x-b y) \frac{\partial h_{n-k}}{\partial x}-x \frac{\partial h_{n-k}}{\partial y}+z \frac{\partial h_{n-k}}{\partial z} .
$$

Proceeding as in (11) using the new variables (7) we only need to consider the terms in (12) for each one of the sums when we let $r=0, \ldots,[k / 2]$. Hence, using equation (10) with $j=k$ we get

$$
\begin{aligned}
& \frac{\partial \tilde{h}_{n-k-1}}{\partial Z} \\
& =-\sum_{r=0}^{[k / 2]} \frac{X}{Y^{3}} \frac{\partial}{\partial Y}\left(\sum _ { l = 2 ( k - 2 r ) } ^ { m - r } c _ { l , r } X ^ { m - l + k - 3 r } \left(\frac{(-1)^{k-2 r}}{(k-2 r) !} A_{k} Y^{2 l-4(k-2 r)} Z^{k-2 r}\right.\right. \\
& \left.\left.\quad+O\left(X^{-1}\right)\right)\right) \\
& \quad-\sum_{r=0}^{[k / 2]} \frac{X^{2}}{Y^{4}} \frac{\partial}{\partial X}\left(\sum _ { l = 2 ( k - 2 r ) } ^ { m - r } c _ { l , r } X ^ { m - l + k - 3 r } \left(\frac{(-1)^{k-2 r}}{(k-2 r) !} A_{k} Y^{2 l-4(k-2 r)} Z^{k-2 r}\right.\right. \\
& \left.\left.\quad+O\left(X^{-1}\right)\right)\right) .
\end{aligned}
$$

and so,

$$
\begin{aligned}
\frac{\partial \tilde{h}_{n-k-1}}{\partial Z} & \sum_{r=0}^{[k / 2]} \sum_{l=2(k-2 r)}^{m-r} c_{l, r} X^{m-l+k-3 r+1}\left(\frac{(-1)^{k+1-2 r}}{(k-2 r) !} A_{k}(2 l-4(k-2 r)\right. \\
& +(m-l+k-3 r)) Y^{2 l-4(k-2 r)-4} Z^{k-2 r}\left(1+O\left(X^{-1}\right)\right) \\
= & \sum_{r=0}^{[k / 2]} \sum_{l=2(k-2 r)}^{m-r} c_{l, r} X^{m-l+k-3 r+1}\left(\frac{(-1)^{k+1-2 r}}{(k-2 r) !} A_{k}(m+l-r-3(k-2 r))\right. \\
& \cdot Y^{2 l-4(k-2 r)-4} Z^{k-2 r}\left(1+O\left(X^{-1}\right)\right) \\
= & \sum_{r=0}^{[k / 2]} \sum_{l=2(k-2 r)}^{m-r} c_{l, r} X^{m-l+k-3 r+1}\left(\frac{(-1)^{k+1-2 r}}{(k-2 r) !} A_{k+1} Y^{2 l-4(k-2 r)-4} Z^{k-2 r}(1\right. \\
& \left.+O\left(X^{-1}\right)\right)
\end{aligned}
$$

Integrating (13) with respect to $Z$ we obtain

$$
\begin{aligned}
\tilde{h}_{n-k-1}= & \sum_{r=0}^{[k / 2]} \sum_{l=2(k-2 r)}^{m-r} c_{l, r} X^{m-l+k-3 r+1}\left(\frac{(-1)^{k+1-2 r}}{(k+1-2 r) !} A_{k+1} Y^{2 l-4(k+1-2 r)}\right. \\
& \cdot Z^{k+1-2 r}\left(1+O\left(X^{-1}\right)\right)+\tilde{g}_{n-k-1}(Y, X) .
\end{aligned}
$$

Since $\tilde{h}_{n-k-1}$ must be invariant by $\tilde{\tau}(p(X, Y, Z))=p(X,-Y, Z)$ and must have dimension $2 m-k-1$ we get that $\tilde{g}_{n-k-1}(X, Y)=\sum_{2 s+2 t=2 m-k-1} \tilde{d}_{s, t} Y^{2 s} X^{t}$. If 
$k$ is even then this is not possible and thus $\tilde{g}_{n-k-1}=0$. Note that in this case $[(k+1) / 2]=[k / 2]$ which implies that

$$
\begin{aligned}
\tilde{h}_{n-k-1}= & \sum_{r=0}^{[(k+1) / 2]} \sum_{l=2(k-2 r)}^{m-r} c_{l, r} X^{m-l+k-3 r+1}\left(\frac{(-1)^{k+1-2 r}}{(k+1-2 r) !} A_{k+1} Y^{2 l-4(k+1-2 r)}\right. \\
& \cdot Z^{k+1-2 r}\left(1+O\left(X^{-1}\right)\right) .
\end{aligned}
$$

Since $\tilde{h}_{n-k-1}$ is a polynomial in $Y$ we must have that it is zero when $l=2(k-2 r)$ and when $l=2(k-2 r)+1$. We emphasize that $A_{k+1} \neq 0$ for $l=2(k-2 r)$ or $l=2(k-2 r)+1$ and thus $c_{l, r}=0$ for $l=2(k-2 r)$ and $l=2(k-2 r)+1$. This implies that $l \geq 2(k+1-2 r)$ which proves (10) for $j=k+1$ and $k$ even. If $k$ is odd then we get that

$$
\tilde{g}_{n-k-1}(X, Y)=\sum_{2 s+2 t=2 m-(k+1)} \tilde{d}_{s, t} Y^{2 s} X^{t}=\sum_{s=0}^{m-(k+1) / 2} d_{s} Y^{2 s} X^{m-s-(k+1) / 2} .
$$

Note that in this case

$$
\begin{aligned}
\tilde{h}_{n-k-1}= & \sum_{r=0}^{[(k+1) / 2]} \sum_{l=2(k-2 r)}^{m-r} c_{l, r} X^{m-l+k-3 r+1}\left(\frac{(-1)^{k+1-2 r}}{(k+1-2 r) !} A_{k+1} Y^{2 l-4(k-2 r)-4}\right. \\
& \cdot Z^{k+1-2 r}\left(1+O\left(X^{-1}\right)\right),
\end{aligned}
$$

where for $r=(k+1) / 2$ we get that $d_{s}=c_{l, r}$. As before, since $\tilde{h}_{n-k-1}$ is a polynomial in $Y$ we must have that it is zero when $l=2(k-2 r)$ and when $l=2(k-2 r)+1$. We emphasize that $A_{k+1} \neq 0$ for $l=2(k-2 r)$ or $l=2(k-2 r)+1$ and thus $c_{l, r}=0$ for $l=2(k-2 r)$ and $l=2(k-2 r)+1$. This implies that $l \geq 2(k+1-2 r)$ which proves (10) for $j=k+1$ with $k$ odd.

It follows from (10) with $r=0$ and $j=0, \ldots, m$ that $b_{l}=0$ for $l=0, \ldots, m$. This implies that $h_{m}$ is a zero which is not possible since $h$ is a first integral. This completes the proof of the theorem.

Now we compute the Darboux polynomials of system (1) that are invariant by $\tau$.

Proposition 11. If $g$ is an irreducible Darboux polynomial for system (1) with cofactor

$$
K=\alpha_{0}+\alpha_{1} x+\alpha_{2} y+\alpha_{3} z
$$

then $f=g \cdot \tau g$ is a Darboux polynomial invariant by $\tau$ with a cofactor of the form

$$
K_{\tau}=2 \alpha_{0}+2 \alpha_{3} z .
$$

Proof. Since system (1) is invariant under $\tau$, then $\tau g$ is a Darboux polynomial of system (1) and with cofactor $\tau(K)$. Moreover, by Lemma $6, g \cdot \tau g$ is also a Darboux polynomial of system (1) with cofactor $K+\tau(K)$. Therefore again by Lemma 6 , the cofactor of $f$ is $K_{\tau}=K+\tau(K)$ which is given in (15).

Proposition 12. System (1) has no Darboux polynomials invariant by $\tau$ with nonzero cofactor $K_{\tau}$. 
Proof. Let $g=g(x, y, z)$ be a Darboux polynomial of system (1) with nonzero cofactor $K_{\tau}$. Then it satisfies

$$
(-a x+b y-y z) \frac{\partial g}{\partial x}+x \frac{\partial g}{\partial y}+\left(-z+y^{2}\right) \frac{\partial g}{\partial z}=2\left(\alpha_{0}+\alpha_{3} z\right) g .
$$

We write $g$ in its homogeneous parts as

$$
g=\sum_{j=0}^{n} g_{j}(x, y, z),
$$

where each $g_{j}$ is a homogeneous polynomial of degree $j$. Computing the terms of degree $n+1$ in (16), we get

$$
L\left(g_{n}\right)=2 \alpha_{3} z g_{n}
$$

Solving it we get

$$
g_{n}=G_{n}\left(y, x y+\frac{z^{2}}{2}\right) e^{\frac{\alpha_{3} z^{2}}{y^{2}}}
$$

where $G_{n}$ is any function in the variables $y$ and $x y+\frac{z^{2}}{2}$. Since $g_{n}$ must be a polynomial we must have $\alpha_{3}=0$. Then we get that $g_{n}$ can be written as in (8). Hence, proceeding exactly as we did in the proof of Theorem 10 taking into account that in the computation of $g_{n-1}$ we have that $2 \alpha_{0} g_{n}$ is in the $O\left(X^{-1}\right)$ we can prove in the same manner that (10) holds. Then, $g_{n}=K_{n}$ a constant and $n=0$. Thus, it follows from (16) that

This concludes the proof.

$$
0=2 \alpha_{0} g_{0} \quad \text { that is } \quad \alpha_{0}=0 .
$$

Proof of Theorem 1. Let $g$ be an irreducible Darboux polynomial of system (1) of degree $n$ and with cofactor $K$ of the form given in (14). Then, from Proposition 11, we can assume that $f=g \cdot \tau g$ is a Darboux polynomial of system (1) and invariant by $\tau$, with degree $2 n$ and non-zero cofactor $K_{\tau}$ of the form in (15). From Proposition 12 , we get that $K_{\tau}=0$. Hence, $f$ must be a polynomial first integral of system (1) that is invariant by $\tau$. By Theorem 10 this is not possible. Note that this proof states for Darboux polynomials with zero and nonzero cofactor.

Now we proceed with the proof of Theorem 3.

Proof of Theorem 3. It follows from Theorems 8 and 1 and Proposition 7 that in order to have a first integral of Darboux type we must have $q$ exponential factors $E_{j}=\exp \left(g_{j}\right)$ with cofactors $L_{j}$ such that $\sum_{j=1}^{q} \mu_{j} L_{j}=0$. Let $G=\sum_{j=1}^{q} \mu_{j} g_{j}$, then $E=\exp (G)$ is an exponential factor of system (1) with cofactor $L=\sum_{j=1}^{q} \mu_{j} L_{j}=0$ (see Lemma 9). So $G$ is a polynomial first integral of system (1). By Theorem 1 this is not possible.

\section{Proof of Theorem 13}

In this section we prove the next result Theorem 13. A corollary of Theorem 13 is Theorem 4

Theorem 13. System (1) has no analytic first integral for the values of the parameters $a, b \in \mathbb{R}$ satisfying:

(i) For all $(a, b)$ such that $a b=0$. 
(ii) For all $(a, b)$ such that $b \leq-a^{2} / 4$.

(iii) For all $(a, b)$ such that $b \neq m(m+a)$ and $m \in \mathbb{N}$.

(iv) For all $(a, b)$ such that $b=m(m+a)$ with $m \in \mathbb{N}$, and either $a \in\left(-\infty, a_{1}(m)\right) \cup$ $\left(a_{2}(m), \infty\right)$ where $a_{1}(m)<a_{2}(m)$ are the unique two real roots of the polynomial $g(a, m)=(8 m-1) a^{4}+\left(8 m^{2}-12 m+2\right) a^{3}+\left(96 m^{2}-12 m-1\right) a^{2}+\left(216 m^{3}-\right.$ $\left.12 m^{2}+8 m\right) a+m^{2}\left(108 m^{2}+8\right)$, or $a \in\left[a_{1}(m), a_{2}(m)\right]$ and $\left\{1, a \sqrt{a^{2}+4 b}\right\}$ is linearly independent on $\mathbb{Z}$.

Before proving Theorem 13 we need an auxiliary lemma that characterizes all the first integrals of the linear part of system (1).

Lemma 14. The linear part of system (1) has the two independent first integrals:

(a) For $a=0$ and $b \neq 0$ the linear part of system (1) has the two independent first integrals $F_{1}=-x^{2}+b y^{2}$ and $F_{2}=z e^{x / b y}$.

(b) For $a \neq 0$ and $b=0$ the linear part of system (1) has the two independent first integrals $F_{1}=x+$ ay and $F_{2}=z e^{y / x}$.

(c) For $a b \neq 0$ and $b=-a^{2} / 4$ the linear part of system (1) has the two independent first integrals $F_{1}=(x+a / 2 y) z^{-a / 2}$ and $F_{2}=z e^{y / x}$.

(d) For $a b \neq 0$ and $b \neq-a^{2} / 4$ the linear part of system (1) has the two independent first integrals $F_{1}=\left(x+\lambda_{2} y\right) z^{\lambda_{3}}$ and $F_{2}=\left(x+\lambda_{3} y\right) z^{\lambda_{2}}$ where $\lambda_{2}$ and $\lambda_{3}$ are the eigenvalues of the linear part of system (1) different from -1 .

Proof. The proof of this lemma follows easily.

Proof of Theorem 13. Case (i.1): $a=0$ and $b \neq 0$. Suppose that $h(x, y, z)=$ $\sum_{k \geq 0} h_{k}(x, y, z)$ is an analytic first integral of system (1) under the conditions of case

(i.1) where $h_{k}$ is the homogeneous part of $h$ of degree $k$ in the $x, y$ and $z$ variables. So $H$ satisfies

$$
(b y-y z) \frac{\partial h}{\partial x}+x \frac{\partial h}{\partial y}+\left(-z+y^{2}\right) \frac{\partial h}{\partial z}=0 .
$$

Note that the homogeneous terms of degree 1 in (17) satisfy the differential equation: $L_{1}\left(h_{1}\right)=0$ where $L_{1}$ is the linear partial differential operator given by $L_{1}=b y \frac{\partial}{\partial x}+x \frac{\partial}{\partial y}-z \frac{\partial}{\partial z}$, i.e. $h_{1}$ is a first integral of the linear vector field $X(x, y, z)=$ $(b y, x,-z)$. As $h_{1}$ is polynomial it follows from Lemma 14 that $h_{1}(x, y, z)=\alpha_{1}\left(-x^{2}+\right.$ $\left.b y^{2}\right)^{l}$. But, as $h_{1}$ is of degree 1 we must have $\alpha_{1}=0$ and so $h_{1} \equiv 0$.

Now the homogeneous terms of degree 2 in (17) satisfy $L_{1}\left(h_{2}\right)=y z \frac{\partial h_{1}}{\partial x}=0$. So, we get $h_{2}=\alpha_{2}\left(-x^{2}+b y^{2}\right)^{l_{2}}$ for some $l_{2} \in \mathbb{N}$. Using the fact that $h_{2}$ is homogeneous of degree 2 we obtain $h_{2}=\alpha_{2}\left(-x^{2}+b y^{2}\right)$. Concerning the terms of degree 3 we have

$$
L_{1}\left(h_{3}\right)=-2 \alpha_{2} x y z
$$

Doing $z=0$ in (18) we have

$$
\text { by } \frac{\partial h_{3}}{\partial x}(x, y, 0)+x \frac{\partial h_{3}}{\partial y}(x, y, 0)=0
$$

and so $h_{3}(x, y, 0)$ is a polynomial first integral of the vector field $X_{3}(x, y)=(b y, x)$. This implies that $h_{3}(x, y, 0)=\hat{\alpha}_{3}\left(-x^{2}+b y^{2}\right)^{l_{3}}$. In order that $h_{3}$ be homogeneous of 
degree 3 we must have $\hat{\alpha}_{3}=0$. So $h_{3}(x, y, z)=z \tilde{h}_{2}(x, y, z)$. Substituting this expression in (18) we get for $z \neq 0$ the equality $L_{1}\left(\tilde{h}_{2}\right)-\tilde{h}_{2}=-2 \alpha_{2} x y$ that has the polynomial solution $\tilde{h}_{2}=\frac{2 \alpha_{2}\left(x^{2}+x y+b y^{2}\right)}{1-4 b}$ if $b \neq 1 / 4$. So $h_{3}=\frac{2 \alpha_{2}\left(x^{2}+x y+b y^{2}\right) z}{1-4 b}$. In a similar way, considering the homogeneous term of degree 4 in (17) we can write

$$
L_{1}\left(h_{4}\right)=\left(y z \frac{\partial h_{3}}{\partial x}-y^{2} \frac{\partial h_{3}}{\partial z}\right)=-\frac{2 \alpha_{2} y\left[y\left(x^{2}+x y+b y^{2}\right)-(2 x+y) z^{2}\right]}{1-4 b} .
$$

This equation admits polynomial solution only if $\alpha_{2}=0$ and, in this case, $h_{4}=$ $\alpha_{4}\left(-x^{2}+b y^{2}\right)^{2}$. All together, until now we have $h_{1}, h_{2}, h_{3} \equiv 0$ and $h_{4}=\alpha_{4}\left(-x^{2}+\right.$ $\left.b y^{2}\right)^{2}$ with $\alpha_{4} \in \mathbb{R}$.

We suppose that we have $h_{1}, h_{2}, \cdots, h_{2 n-1} \equiv 0$ and $h_{2 n}=\alpha_{2 n}\left(-x^{2}+b y^{2}\right)^{n}$ with $\alpha_{2 n} \in \mathbb{R}$. So, the terms of degree $2 n+1$ satisfy

$$
L_{1}\left(h_{2 n+1}\right)=y z \frac{\partial h_{2 n}}{\partial x}=-2 n \alpha_{2 n} x y z\left(-x^{2}+b y^{2}\right)^{n-1} .
$$

As before this implies that $h_{2 n+1}(x, y, 0)=\tilde{\alpha}_{2 n+1}\left(-x^{2}+b y^{2}\right)^{l_{2 n+1}}$. This means that $h_{2 n+1}=z \tilde{h}_{2 n}$ where $\tilde{h}_{2 n}$ satisfies

$$
L_{1}\left(\tilde{h}_{2 n}\right)-\tilde{h}_{2 n}=-2 \alpha_{2 n} x y\left(-x^{2}+b y^{2}\right)^{n-1} .
$$

This means that $h_{2 n+1}(x, y, 0)=\tilde{\alpha}_{2 n+1}\left(-x^{2}+b y^{2}\right)^{l_{2 n+1}}$ that implies that $h_{2 n+1}(x, y, 0)=$ $z \tilde{h}_{2 n}$ where $\tilde{h}_{2 n}$ satisfies

$$
L\left(\tilde{h}_{2 n}\right)-\tilde{h}_{2 n}=-2 \alpha_{2 n} x y\left(-x^{2}+b y^{2}\right)^{n-1},
$$

that admits polynomial solutions just when $\alpha_{2 n}=0$ and, in this case, $\tilde{h}_{2 n}=$ $\frac{2 n \alpha_{2 n}\left(-x^{2}+b y^{2}\right)^{n-1}\left(x^{2}+x y+b y^{2}\right)}{1-4 b}$ and $h_{2 n+1}=\frac{2 n \alpha_{2 n}\left(-x^{2}+b y^{2}\right)^{n-1}\left(x^{2}+x y+b y^{2}\right) z}{1-4 b}$.

For the homogeneous terms of degree $2 n+2$ of (17) we have

$$
L\left(h_{2 n+2}\right)=y z \frac{\partial h_{2 n+1}}{\partial x}-y^{2} \frac{\partial h_{2 n+1}}{\partial z}
$$

that has polynomial solution just when $\alpha_{2 n}=0$ and the homogeneous solution of degree $2 n+2$ is given by $h_{2 n+2}=\alpha_{2 n+1}\left(-x^{2}+b y^{2}\right)^{n+1}$. So we have by induction under the degree of homogeneity that $h_{k} \equiv 0$ for all $k \geq 1$ and system (1) restricted to case (i.1) with $b \neq 1 / 4$ has no analytic first integral.

Case (i.1.1): $a=0$ and $b=1 / 4$. Suppose that $h(x, y, z)=\sum_{k \geq 0} h_{k}(x, y, z)$ is an analytic first integral of system (1) under the conditions of case (i.1.1) where $h_{k}$ is the homogeneous part of $h$ of degree $k$ in the $x, y$ and $z$ variables. So $h$ satisfies

$$
\left(\frac{y}{4}-y z\right) \frac{\partial h}{\partial x}+x \frac{\partial h}{\partial y}+\left(-z+y^{2}\right) \frac{\partial h}{\partial z}=0 .
$$

Note that the homogeneous terms of degree 1 in (19) satisfy the differential equation: $L_{2}\left(h_{1}\right)=0$ where $L_{2}$ is the linear partial differential operator given by $L_{2}=\frac{y}{4} \frac{\partial}{\partial x}+x \frac{\partial}{\partial y}-z \frac{\partial}{\partial z}$, i.e. $h_{1}$ is a first integral of the linear vector field $X(x, y, z)=$ $\left(\frac{y}{4}, x,-z\right)$. As $h_{1}$ is polynomial it follows from Lemma 14 that $h_{1}(x, y, z)=\alpha_{1}\left(4 x^{2}-\right.$ $\left.y^{2}\right)^{l}$. But, as $h_{1}$ is of degree 1 we must have $\alpha_{1}=0$ and so $h_{1} \equiv 0$. 
Now the homogeneous terms of degree 2 in (19) satisfy $L_{2}\left(h_{2}\right)=y z \frac{\partial h_{1}}{\partial x}=0$. So, we get $h_{2}=\alpha_{2}\left(4 x^{2}-y^{2}\right)^{l_{2}}$ for some $l_{2} \in \mathbb{N}$. Using the fact that $h_{2}$ is homogeneous of degree 2 we obtain $h_{2}=\alpha_{2}\left(4 x^{2}-y^{2}\right)$. Concerning the terms of degree 3 we have

$$
L_{2}\left(h_{3}\right)=-2 \alpha_{2} x y z \text {. }
$$

This equation has polynomial solution only when $\alpha_{2}=0$ and, in this case, $h_{3}=$ $\alpha_{3}\left(4 x^{2}-y^{2}\right)^{l_{3}}$ for some $l_{3} \in \mathbb{N}$. But as $h_{3}$ is homogeneous of degree 3 we get $\alpha_{3}=0$ and so $h_{3} \equiv 0$. Now using this fact the homogeneous terms of degree 4 in (19) satisfy

$$
L_{2}\left(h_{4}\right)=0,
$$

that have homogeneous polynomial solution of degree 4 of the form $h_{4}=\alpha_{4}\left(4 x^{2}-\right.$ $\left.y^{2}\right)^{2}$ with $\alpha_{4} \in \mathbb{R}$.

Suppose that we have $h_{1}, h_{2}, \cdots, h_{2 n-1} \equiv 0$ and $h_{2 n}=\alpha_{2 n}\left(-x^{2}+b y^{2}\right)^{n}$ with $\alpha_{2 n} \in \mathbb{R}$. So, the terms of degree $2 n+1$ satisfy

$$
L_{2}\left(h_{2 n+1}\right)=y z \frac{\partial h_{2 n}}{\partial x}=-2 n \alpha_{2 n} x y z\left(-x^{2}+b y^{2}\right)^{n-1} .
$$

Then the homogeneous terms of degree $2 n+1$ satisfy

$$
L_{2}\left(h_{2 n+1}\right)=y z \frac{\partial h_{2 n}}{\partial x}=8 n \alpha_{2 n} x y z\left(4 x^{2}-y^{2}\right)^{n-1}
$$

that has polynomial solution only if $\alpha_{2 n}=0$ and, in this case, the homogeneous polynomial solution of this system is given by $h_{2 n+1}=\alpha_{2 n+1}\left(4 x^{2}-y^{2}\right)^{n+1}$. So we have by induction under the degree of homogeneity that $h_{k} \equiv 0$ for all $k \geqslant 1$ and system (1) restricted to case (i.1.1) has no analytic first integral.

Case (i.2): $a \neq 0$ and $b=0$. If $h(x, y, z)=\sum_{k \geq 0} h_{k}(x, y, z)$ is an analytic first integral of system (1) under the conditions of case (i.2) then $H$ satisfies

$$
-(a x+y z) \frac{\partial h}{\partial x}+x \frac{\partial h}{\partial y}+\left(-z+y^{2}\right) \frac{\partial h}{\partial z}=0 .
$$

The terms of degree 1 in (20) satisfy $L_{3}\left(h_{1}\right)=0$ where $L_{3}$ is the linear partial differential operator

$$
L_{3}=-a x \frac{\partial}{\partial x}+x \frac{\partial}{\partial y}-z \frac{\partial}{\partial z} .
$$

From $L_{3}\left(h_{1}\right)=0$ if follows from Lemma 14 that $h_{1}(x, y, z)=\alpha_{1}(x+a y)$.

Now the homogeneous terms of degree 2 satisfy $L_{2}\left(h_{2}\right)=y z \frac{\partial h_{1}}{\partial x}-y^{2} \frac{\partial h_{1}}{\partial z}=\alpha_{1} y z$ that has the solution

$$
h_{2}(x, y, z)=-\frac{\alpha_{1}(x+y+a z) z}{1+a}+\alpha_{2}(x+a y)^{2}+\beta_{2} x^{-1 / a} z,
$$

when $a \neq-1$. Observe that the part that depends on $\beta_{2}$ is polynomial only for $a=-1 / n$ with $n \in \mathbb{N}$.

Suppose that $a \neq-1 / n$ with $n \in \mathbb{N}$. So $\beta_{2}$ must be zero and the homogeneous terms of degree 3 in (20) satisfy

$$
L_{3}\left(h_{3}\right)=y z \frac{\partial h_{2}}{\partial x}-y^{2} \frac{\partial h_{2}}{\partial z}
$$


that has polynomial solution only when $\alpha_{1}=0$ and, in this case,

$$
h_{3}=-\frac{2 \alpha_{2}(x+a y)(x+(1+a) y) z}{a^{2}(1+a)}+\alpha_{3}(x+a y)^{3} .
$$

In a similar way the homogeneous terms of degree 4 are solutions of the partial differential equation

$$
L_{3}\left(h_{2}\right)=y z \frac{\partial h_{3}}{\partial x}-y^{2} \frac{\partial h_{3}}{\partial z}
$$

that are polynomial. The solution exists only for $\alpha_{2}=0$ and is given by

$$
h_{4}=-\frac{3 \alpha_{3}(x+a y)^{2}(x+(1+a) y) z}{1+a}+\alpha_{4}(x+a y)^{4} .
$$

Now suppose by induction that

$$
h_{n}=-\frac{(n-1) \alpha_{n-1}(x+a y)^{n-2}(x+(1+a) y) z}{1+a}+\alpha_{n}(x+a y)^{n}
$$

with $n \geq 4$. Then the homogeneous terms of degree $n+1$ satisfy $L_{3}\left(h_{n+1}\right)=$ $y \frac{\partial h_{n}}{\partial x}-y^{2} \frac{\partial h_{n}}{\partial z} z$ that just admit polynomial solutions if $\alpha_{n-1}=0$ and, in this case, we have

$$
h_{n+1}=-\frac{n \alpha_{n}(x+a y)^{n-1}(x+(1+a) y) z}{1+a}+\alpha_{n+1}(x+a y)^{n+1} .
$$

So, $h_{k} \equiv 0$ for all $k \geq 1$ and system (1) has no analytic first integral restricted to the case (i.2) when $a \neq-1 / n$.

Case (i.2.1): $a=-1$ and $b=0$. Suppose that $h=\sum_{k \geq 1} h_{k}$ is an analytic first integral of system (1) with $a=-1$ and $b=0$. So $h$ satisfies

$$
(x-y z) \frac{\partial h}{\partial x}+x \frac{\partial h}{\partial y}+\left(-z+y^{2}\right) \frac{\partial h}{\partial z}=0 .
$$

The homogeneous terms of degree 1 satisfy $L_{4}\left(h_{1}\right)=0$, where $L_{4}=x \frac{\partial}{\partial x}+$ $x \frac{\partial}{\partial y}-z \frac{\partial}{\partial z}$. The characteristic equations associated to the partial differential equation $L_{4}\left(h_{1}\right)=0$ are

$$
\frac{d y}{d x}=1, \quad \frac{d z}{d x}=-\frac{z}{x} .
$$

They have the general solutions $x-y=c_{1}$ and $z x=c_{2}$, where $c_{1}$ and $c_{2}$ are constants of integration. So doing the change of coordinates

$$
u=x-y, \quad v=x z, \quad w=y,
$$

the equation $L_{4}\left(h_{1}\right)=0$ is written as

$$
(u+w) \frac{d \bar{h}_{1}}{d w}=0 .
$$

This implies that $\bar{h}_{1}(u, v, w)=g_{1}(u, v)=g_{1}(x-y, x z)$, and as $h_{1}$ has degree 1 we obtain $h_{1}(x, y, z)=\alpha_{1}(x-y)$.

The homogeneous terms of degree 2 in (21) satisfy

$$
L_{4}\left(h_{2}\right)=y z \frac{\partial h_{1}}{\partial x}-y^{2} \frac{\partial h_{1}}{\partial z}=\alpha_{1} y z .
$$


In the new coordinates $u, v, w$ this last equation becomes

$$
(u+w) \frac{d \bar{h}_{2}}{d w}=\frac{\alpha_{1} v w}{u+w} .
$$

Therefore $\bar{h}_{2}(u, v, w)=\alpha_{1} v\left(\frac{u}{u+w}+\log (u+w)\right)+\bar{f}_{2}(u, v)$. Going back to the $x, y, z$ coordinates we have $h_{2}(x, y, z)=\alpha_{1} z(x-y+x \log (x))+f_{2}(x-y, x z)$. As $h_{2}$ is a homogeneous polynomial of degree 2 we have $\alpha_{1}=0$ and $h_{2}(x, y, z)=\sum_{i=0}^{1} \alpha_{2, i}(y-$ $x)^{2-2 i}(x z)^{i}$.

Performing with the homogeneous terms of degree 3 in the same way than before we obtain

$$
L_{4}\left(h_{3}\right)=y z \frac{\partial h_{2}}{\partial x}-y^{2} \frac{\partial h_{2}}{\partial z} .
$$

This has polynomial solution only when $\alpha_{2,0}=0$, and considering that $h_{3}$ is homogeneous of degree 3 we have

$$
h_{3}=-\frac{\alpha_{2,1}}{6}\left(2 y^{2}+3(x+y) z^{2}\right)+\sum_{i=0}^{i} \alpha_{3, i}(y-x)^{3-2 i}(x z)^{i} .
$$

Solving (21) with respect to the homogeneous terms of degree 4 we must solve

$$
L_{4}\left(h_{4}\right)=y z \frac{\partial h_{3}}{\partial x}-y^{2} \frac{\partial h_{3}}{\partial z} .
$$

This has a polynomial solution only if $\alpha_{2,1}=\alpha_{3,0}=\alpha_{3,1}=0$ and, in this case, the homogeneous part of degree 4 is $h_{4}=\sum_{i=0}^{2} \alpha_{4, i}(y-x)^{4-2 i}(x z)^{i}$.

Taking into account the expression of $h_{4}$ and solving the homogeneous part of (21) of degree 5 we obtain

$$
h_{5}=-\frac{\alpha_{4,2}}{3} x z\left(2 y^{3}+3(x+y) z^{2}\right)+\sum_{i=0}^{2} \alpha_{5, i}(y-x)^{5-2 i}(x z)^{i} .
$$

We consider, by induction that for fixed $n \geq 1$ we have

$$
h_{2 n-1}=-\frac{(n-1) \alpha_{2(n-1), n-1}}{3}(x z)^{n-2}\left(2 y^{3}+3(x+y) z^{2}\right)+\sum_{i=0}^{n-1} \alpha_{2 n-1, i}(y-x)^{2(n-i)-1}(x z)^{i}
$$

and

$$
h_{2 n}=\sum_{i=0}^{n} \alpha_{2 n, i}(y-x)^{2 n-1}(x z)^{i} .
$$

So the homogeneous terms of degree $2 n+1$ in (21) satisfy

$$
L_{4}\left(h_{2 n+1}\right)=y z \frac{\partial h_{2 n}}{\partial x}-y^{2} \frac{\partial h_{2 n}}{\partial z} .
$$

This has a polynomial solution only when $\alpha_{2 n, j}=0$ for $j=0, \cdots n-1$ and, in this case, we get

$$
h_{2 n+1}=-\frac{n \alpha_{2 n, n}}{3}(x z)^{n-1}\left(2 y^{3}+3(x+y) z^{2}\right)+\sum_{i=0}^{n} \alpha_{2 n+1, i}(y-x)^{2(n-i)+1}(x z)^{i} .
$$


The homogeneous terms of degree $2 n+2$ satisfy

$$
L_{4}\left(h_{2 n+2}\right)=y z \frac{\partial h_{2 n+1}}{\partial x}-y^{2} \frac{\partial h_{2 n+1}}{\partial z} .
$$

This has a polynomial solution only when $\alpha_{2 n, n}=\alpha_{2 n+1, j}=0$ for $j=0, \cdots n$, and, we have

$$
h_{2 n+2}=\sum_{i=0}^{n+1} \alpha_{2 n+2, i}(y-x)^{2(n+1-i)}(x z)^{i} .
$$

All together we can conclude that $H$ is constant and so system (1) has no analytical first integral restricted in the case (i.2.1).

Case (i.2.2): $a=-1 / n$ with $n>1$ and $b=0$. Suppose that $h(x, y, z)=\sum_{k \geq 0} h_{k}(x, y, z)$ is an analytic first integral of system (1) under the conditions of case (i.2.1). Then $H$ satisfy

$$
\left(-\frac{x}{n}-y z\right) \frac{\partial h}{\partial x}+x \frac{\partial h}{\partial y}+\left(-z+y^{2}\right) \frac{\partial h}{\partial z}=0 .
$$

The terms of degree 1 in (23) satisfy $L_{5}\left(h_{1}\right)=0$ where $L_{5}$ is the linear partial differential operator given by

$$
L_{5}=\frac{x}{n} \frac{\partial}{\partial x}+x \frac{\partial}{\partial y}-z \frac{\partial}{\partial z} .
$$

The characteristic equations associated to the partial differential equation $L_{5}\left(h_{1}\right)=$ 0 are

$$
\frac{d y}{d x}=n, \quad \frac{d z}{d x}=-\frac{n z}{x} .
$$

They have the general solution $y-n x=c_{1}$ and $x^{n} z=c_{2}$ where $c_{1}$ and $c_{2}$ are constants of integration. So doing the change of coordinates

$$
u=y-n x, \quad v=x^{n} z \quad w=y,
$$

whose inverse change is

$$
x=\frac{-u+v}{n}, \quad z=\frac{n^{n} v}{(-u+v)^{n}}, \quad y=w,
$$

the equation $L_{5}\left(h_{1}\right)=0$ becomes

$$
\frac{(-u+w)}{n} \frac{d \bar{h}_{1}}{d w}=0
$$

its solution is $\bar{h}_{1}(u, v, w)=g_{1}(u, v)=g_{1}\left(y-n x, x^{n} z\right)$. As $h_{1}$ has degree 1 we obtain $h_{1}(x, y, z)=\alpha_{1}(y-n x)$.

Now the homogeneous terms of degree 2 in (23) satisfy

$$
L_{5}\left(h_{2}\right)=y z \frac{\partial h_{1}}{\partial x}-y^{2} \frac{\partial h_{1}}{\partial z}=-n \alpha_{1} y z .
$$

In the new coordinates $u, v, w$ it becomes

$$
\frac{d \bar{h}_{2}}{d w}=-\frac{n^{n+1} \alpha_{1} v w}{(-u+w)^{n-1}} .
$$

I ts solution is

$$
\bar{h}_{2}(u, v, w)=-\frac{n^{n+1} \alpha_{1} v(u-n w)}{(n-1)(-v+w)^{n}}+\bar{f}_{2}(u, v) .
$$


In the $x, y, z$ coordinates we have

$$
h_{2}(x, y, z)=\frac{n \alpha_{1} z(n x+(n-1) y)}{n-1}+f_{2}\left(x-n y, x^{n} z\right) .
$$

As $h_{2}$ is a polynomial homogeneous of degree 2 we conclude that

$$
h_{2}(x, y, z)=\frac{n \alpha_{1} z(n x+(n-1) y)}{n-1}+\alpha_{2}(y-n x)^{2} .
$$

The homogeneous terms of degree 3 satisfy

$$
L_{5}\left(h_{3}\right)=y z \frac{\partial h_{2}}{\partial x}-y^{2} \frac{\partial h_{2}}{\partial z},
$$

that in the $u, v, w$ coordinates is written in the form

$$
\frac{d \bar{h}_{3}}{d w}=\frac{n^{2} w(-u+w)\left(-u w+n\left(w^{2}-n^{2 n} v^{2}(-u+w)^{-2 n}\right)\right)}{(n-1)}-\frac{2 \alpha_{2} n^{n+2} u v}{(-u+w)^{n+1}} .
$$

It has polynomial solutions only for $\alpha_{1}=0$ and, in this case, the homogeneous solution of degree 3 written in coordinates $x, y, z$ is of the form

$$
h_{3}=\frac{2 n \alpha_{2} z(y-n x)(n x+(n-1) y)}{n-1}+\alpha_{3}(n x-y)^{3} .
$$

Suppose by induction that $h_{m}(x, y, z)=\frac{(m-1) n \alpha_{m-1}(y-n x)^{m-2}(n x-(n-1) y)}{n-1}+$ $\alpha_{m}(y-n x)^{m}$ and $m<n+1$. Solving equation (23) with respect to the terms of degree $m+1$ we must solve

$$
L_{5}\left(h_{m+1}\right)=y z \frac{\partial h_{m}}{\partial x}-y^{2} \frac{\partial h_{m}}{\partial z} .
$$

They can be solved using the coordinates $u, v$ and $w$ in the same way than before and its solution is polynomial only if $\alpha_{m-1}=0$ and

$$
h_{m+1}=\frac{m n \alpha_{m} z(y-n x)^{m-1}(n x+(n-1) y)}{n-1}+\alpha_{m+1}(y-n x)^{m+1} .
$$

We can do this for all $m<n+1$ (this is due to the degree of the polynomial $x^{n} z$ ), and we conclude that $h_{k} \equiv 0$ for all $k<n$.

Now, for the homogeneous part of $h$ of degree $k \geq n$ we have

$$
h_{n}=\frac{(n-1) n \alpha_{n-1} z(y-n x)^{n-2}(n x+(n-1) y)}{n-1}+\alpha_{n}(y-n x)^{n} .
$$

Solving (23) with respect to the homogeneous terms of degree $n+1$ we obtain polynomial solutions only if $\alpha_{n-1}=0$, and this is

$$
h_{n+1}=\frac{n^{2} \alpha_{n} z(y-n x)^{n-1}(n x+(n-1) y)}{n-1}+\sum_{i=0}^{1} \alpha_{n+1, i}(y-n x)^{(n+1)(1-i)}\left(x^{n} z\right)^{i} .
$$

In order to find the homogeneous part of degree $n+2$ satisfying (23) we have to solve

$$
L_{5}\left(h_{n+2}\right)=y z \frac{\partial h_{n+1}}{\partial x}-y^{2} \frac{\partial h_{n+1}}{\partial z} .
$$

Note that when we consider this partial differential equation in the $u, v, w$ coordinates as before the left hand part of the resultant ordinary differential equation is $\frac{(-u+w)}{n} \frac{d \bar{h}_{n+2}}{d w}$, and the right hand part is composed of two parts. The first part is 
the term $\frac{n^{2} \alpha_{n} z(y-n x)^{n-1}(n x+(n-1) y)}{n-1}$ in the new coordinates, and the second part is $\sum_{i=0}^{1} \alpha_{n+1, i}(y-n x)^{(n+1)(1-i)}\left(x^{n} z\right)^{i}$ also in the new coordinates. Moreover, by the linearity of the integral which provides the solution of this ordinary differential equation, we can study these two parts separately.

Note that the first part has the same form than in the induction process up to $n$. So we can conclude that the next step of the iterative process forces that this term must vanish. So we just have to study the second part. For these terms we have to study the expression obtained after integrating. But when we integrate, in the iterative process terms of the form $\omega(y-n x)^{r}\left(x^{n} z\right)^{s}$, we obtain two new terms which are

$$
\omega \frac{s x^{n}(y-n x)^{r}\left[2 n^{2} x^{2}-2 n(n+2) x y+(n+1)(n+2) y^{2}\right]\left(x^{n} z\right)^{s-1}}{(n+1)(n+2)}
$$

and

$$
\begin{aligned}
& \omega \frac{x^{n-1}(y-n x)^{r-1} n\left(n^{2} s+(r-s) n+r\right) x^{2} z^{2}\left(x^{n} z\right)^{s-1}}{(n+1)(n-1)}+ \\
& \omega \frac{x^{n-1}(y-n x)^{r-1}\left[(n-1)\left(n^{2} s+(r-s) n+r\right) x y-n(n-1) s y^{2}\right] z^{2}\left(x^{n} z\right)^{s-1}}{(n+1)(n-1)} .
\end{aligned}
$$

These two new terms will be integrated again in the next step providing two new terms that are not polynomial if $n(n+2)\left(3 s n^{2}+n(s-r)-r-2\right) \neq 0$ and $(n-$ 1) $\left(3 s n^{2}+n(s-r)-r\right) \neq 0$, respectively. As there is no pair $(r, s) \in \mathbb{N}^{2}$ such that these two expressions simultaneously vanish we must have $\omega=0$, and so no term of the form $\omega(y-n x)^{r}\left(x^{n} z\right)^{s}$ can be present in the analytic first integral. This argument, applied to each step of the iterative process implies that there is no analytic first integral for system (1) restricted to the conditions of case (i.2.2).

Case (ii): $a b \neq 0$ and $b \leq-a^{2} / 4$. First consider $b=-a^{2} / 4$. From Lemma 14 the linear part of system (1) has the first integral $F_{1}=(x+a / 2 y) z^{-a / 2}$ which is polynomial when $-\frac{a}{2} \in \mathbb{N}$, i.e. $b=-k^{2}$ with $k=a / 2$. In this case the vector field associated to system (1) has equilibria $x_{0}=(0,0,0)$ and $x_{ \pm}=\left(0, \pm i k,-k^{2}\right) \in \mathbb{C}^{3}$. Moreover the eigenvalues associated to the equilibria at the origin are $\lambda_{1}=-1$ and $\lambda_{2,3}=\frac{1 \pm \sqrt{1-4 k^{2}}}{2} \in \mathbb{C} \backslash \mathbb{R}$. So, system (1) cannot have analytic first integral in a neighbourhood of the origin, because this equilibrium is hyperbolic and by the Hartmann Theorem in a surface it has a focal behaviour.

Now, for $b<-a^{2} / 4$ we observe here that the same argument can be applied to this case. Then the eigenvalues at the origin are: $\lambda_{1}=-1$ and $\lambda_{2,3}=\frac{-a \pm \sqrt{a^{2}+4 b}}{2} \in \mathbb{C} \backslash \mathbb{R}$.

Case (iii)-(iv): $a \neq 0$ and $b>0$. In this case system (1) has as equilibria $x_{0}=$ $(0,0,0) \in \mathbb{R}^{3}$ and $x_{ \pm}=(0, \pm \sqrt{b}, b) \in \mathbb{R}^{3}$. Also the linearisation at the origin has eigenvalues $\lambda_{1}=-1$ and $\lambda_{2,3}=\frac{-a \pm \sqrt{a^{2}+4 b}}{2}$. We know that a necessary condition in order that system (1) has a analytic first integral is that its linear part $X_{0}(x, y, z)=$ $(-a x+b y, x,-z)$ admits a polynomial first integral. 
From Lemma 14 in this case the linear part of system (1) has two independent first integrals given by $F_{1}(x, y, z)=\left(x-\lambda_{3} y\right) z^{\lambda_{2}}$ and $F_{2}(x, y, z)=\left(x-\lambda_{2} y\right) z^{\lambda_{3}}$. This means that $X_{0}$ has a polynomial first integral just if $\lambda_{2} \in \mathbb{N}$ or $\lambda_{3} \in \mathbb{N}$.

Suppose for instance that $\lambda_{2}=m \in \mathbb{N}$. So $b=m(m+a)$.

In accordance with [7] another necessary condition in order that system (1) has a analytic first integral is that there exist $m_{1}, m_{2}, m_{3} \in \mathbb{N}$ not all zero such that $\sum_{i=1}^{3} m_{i} \lambda_{i}=0$. So, in order that system (1) can have an analytic first integral the set $\left\{1, a, \sqrt{a^{2}+4 b}\right\}$ must be linearly dependent on $\mathbb{Z}$.

Now under these hypotheses let $p_{+}(\lambda)$ be the characteristic polynomial associated to the equilibrium $x_{+}$. Consider $m \in \mathbb{N}$ fixed in $b=m(m+a)$. We have $p_{+}(\lambda)=$ $\lambda^{3}+(a+1) \lambda_{2}+a \lambda+2 m(a+m)$.

Note that $p_{+}(\lambda)$ has discriminant $g(a, m)=(8 m-1) a^{4}+\left(8 m^{2}-12 m+2\right) a^{3}+$ $\left(96 m^{2}-12 m-1\right) a^{2}+\left(216 m^{3}-12 m^{2}+8 m\right) a+m^{2}\left(108 m^{2}+8\right)$. Moreover $p_{+}(\lambda)$ has three real roots if and only if $g(a, m)<0$ (see [4]). It is easy to see that this polynomial has two real roots in $a_{1}(m)$ and $a_{2}(m)$ with $-(m+1)<a_{1}(m)<-m<$ $a_{2}(m)<0$. So, if $a \in\left(-\infty, a_{1}(m)\right) \cup\left(a_{2}(m), \infty\right)$ we have $g(a, m)>0$ if follows that for these values of $a$ the characteristic polynomial $p_{+}(\lambda)$ has two complex roots and hence system (1) has no analytic first integral. This completes the proof of statement (iv) of Theorem (13).

\section{ACKNOWLEDGEMENTS}

The first author is partially supported by Fapesp grant number 2013/15941-5. The second author is partially supported by a MINECO/FEDER grant MTM200803437, a CIRIT grant number 2009SGR-410, by ICREA Academia, and grant FP7PEOPLE-2012-IRSES 318999, and FEDER/UNAB10-4E-378. Both, first and second authors, are also supported by the joint project CAPES-MECD grant PHB2009-0025-PC and by FP7-PEOPLE-2012-IRSES 316338. The third author has been supported by FCT (grant PTDC/MAT/117106/2010 and through CAMGSD).

\section{REFERENCES}

[1] C. Christopher, J. Llibre And J.V. Pereira, Multiplicity of invariant algebraic curves in polynomial vector fields, Pacific J. Math. 229 (2007), 63-117.

[2] G. Darboux, Mèmoire sur les équations différentielles algébraiques du premier ordre et du premier degré (Métanges), Bull. Sci. Math. 2éme série 2 (1878), 60-96; 123-144; 151-200.

[3] F. Dumortier, J. Llibre And J.C. Artés, Qualitative theory of planar differential systems, Universitext, Springer-Verlag, New York, 2006.

[4] R.S. IRving, Integers, polynomials and rings. Springer-Verlag. New York. 2009.

[5] J. LliBRe AND X. ZhANG, On the Darboux integrability of the polynomial differential systems, Qualitative Theory and Dynamical Systems 11 (2012), 129-144.

[6] J. LlibRe And X. Zhang, Darboux theory of integrability for polynomial vector fields in $\mathbb{R}^{n}$ taking into account the multiplicity at infinity, Bull. Sci. Math. 133 (2009), 765-778.

[7] W. Li, J. LliBre AND X. Zhang, Local first integrals of differential systems and diffeomorphisms, A. Angew. Math. Phys. 54 (2003), 235-255.

[8] Z. Hu, M. Aldazharova, T.M. Aldibekov and V. Romanovski, Integrability of 3-dim polynomial systems with three invariant planes, Nonlinear Dynamics 74 (2013), 1077-1092.

[9] A.M. Rucklidge, Chaos in models of double convection, J. Fluid Mech. 237 (1992), 209-229.

[10] N. Svetoslav, First Lyapunov Value and Bifurcation Behavior of Specific Class of ThreeDimensional Systems, Int. J. bifurcation Chaos 14 (2004), 2811-2823. 
[11] X. WANG, Si'lnikov chaos and Hopf bifurcation analysis of Rucklidge system, Chaos, Solitions and Fractals 42 (2009), 2208-2217.

1 Centro de Matematica ComputaÇão e Cognição. Universidade Federal do ABC, 09210-170. SAnto AndRE. S.P. BraziL

E-mail address: mauricio.lima@ufabc.edu.br

2 Departament de Matemàtiques, Universitat Autònoma de Barcelona, 08193 Bellaterra, Barcelona, Catalonia, Spain

E-mail address: jllibre@mat.uab.cat

3 Departamento de Matemática, Instituto Superior Técnico, Universidade Técnica De Lisboa, Av. Rovisco Pais 1049-001, Lisboa, Portugal

E-mail address: cvalls@math.ist.utl.pt 